\title{
Evaluation of Heater Temperature to Detect Gases in a Flowing Fluid
}

\author{
Christoph J. Hepp ${ }^{1}$, Florian T. Krogmann ${ }^{1}$, Gerald A. Urban ${ }^{2}$ \\ ${ }^{1}$ Innovative Sensor Technology IST AG, Stegruetistrasse 14, 9642 Ebnat-Kappel, Switzerland, \\ christoph.hepp@ist-ag.com \\ ${ }^{2}$ Laboratory for Sensors, Department of Microsystems Engineering - IMTEK, University of Freiburg, \\ Georges-Koehler-Allee 103, 79110 Freiburg, Germany
}

\begin{abstract}
:
In this contribution the behavior of a calorimetric flow sensor was investigated, at which only the temperature of the heater is evaluated. A time-dependent excitation ( $f=1 \mathrm{~Hz}$ ) was applied to the heating element with a constant power amplitude. The influence of different gases and flow velocities on the heater temperature were observed. Measurements and simulations were carried out in no-flow condition and in flowing fluids in order to obtain the restrictions regarding to a simultaneous detection of flow speed and gas type. A simultaneous measurement of flow velocity and gas type was possible, however certain conditions had to be respected, e.g. a high difference in thermal conductivity which is predetermined by the flow speed range.
\end{abstract}

Key words: Thermal Flow Sensor, Heater Temperature, Flowing Fluid, Detection of Gas Type, Time Modulated Excitation.

\section{Introduction}

Thermal flow sensors are well-known in the market for many decades [1]. Low power consumption, low production costs and a small size make them suitable for several applications like process control or breathing gas systems [2, 3].

Thermal flow sensors differ in the used measurement principle. Chips, where the conductometric principle is used, feature usually one heater and one temperature sensor. Two operating modes are established: constant temperature anemometer (CTA) or constant current anemometer (CCA).

The calorimetric principle features a heat source embedded between two temperature sensors in a few hundred micrometers distance. For an improvement in response time and sensitivity, these elements are often located on a membrane [4].

All these types of sensors require calibration prior to performing flow measurements.

The measuring characteristic of thermal flow sensors is strongly affected by the thermal gas properties. Therefore, the elements have to be calibrated to the specific gas targeted in order to obtain an accurate flow speed measurement. If the fluid or composition is changing, recalibrations or correction factors, containing the thermal gas properties, have to be implemented in the system. This will overcome the drawback, but on the other hand increase costs and maintenance procedures.

Table 1 shows the main thermal properties of several gases. It illustrates that especially the thermal conductivity of the gas can vary in a wide range.

Tab. 1: Thermal gas properties at a temperature of $25^{\circ} \mathrm{C}$ and a pressure of 1 bar [5].

\begin{tabular}{|c|c|c|c|}
\hline Gas type & $\begin{array}{c}\text { Thermal } \\
\text { conductivity } \\
{[\mathrm{W} /(\mathrm{m} \cdot \mathrm{K})]}\end{array}$ & $\begin{array}{c}\text { Specific } \\
\text { heat } \\
\text { capacity } \\
{[\mathrm{J} /(\mathrm{g} \cdot \mathrm{K})]}\end{array}$ & $\begin{array}{c}\text { Density } \\
{\left[10^{-3}\right.} \\
\left.\mathrm{g} / \mathrm{cm}^{3}\right]\end{array}$ \\
\hline $\mathrm{Air}$ & 0.0260 & 1.006 & 1.169 \\
\hline $\mathrm{Ar}$ & 0.0178 & 0.522 & 1.612 \\
\hline $\mathrm{CO}_{2}$ & 0.0169 & 0.851 & 1.785 \\
\hline $\mathrm{He}$ & 0.1548 & 5.147 & 0.162 \\
\hline $\begin{array}{c}\mathrm{Ar}-\mathrm{He}= \\
50 \%: 50 \%\end{array}$ & 0.0605 & 0.946 & 0.911 \\
\hline
\end{tabular}

Thermal conductivity sensors are well established on the market for years and are very suited to analyze gas compositions under no-flow conditions [6]. Several research groups 
showed it was possible to measure flow speed and thermal conductivity of the gas with a thermal flow sensor based on the calorimetric principle. Therefore, time modulated excitation was used instead of the widely-used DCexcitation. An assumption thereby is that the flow rate has to be constrained to a certain flow range or has to be known $[7,8]$.

Hence, the gas independent flow measurement with a thermal flow sensor is still a challenge. In this context, we used time dependent heating of a resistor to detect flow speed and gas type.

This paper aims at defining what flow speed and thermal properties have to be met to obtain a simultaneous gas type and flow speed measurement. Only the heater temperature of a calorimetric flow sensor was considered in this investigation.

Beside flow speed various gases have been tested to characterize the dominant physical parameter.

\section{Sensor Chip Description}

Figure 1 shows the top view of the sensor's layout.

The chip was fabricated on a glass substrate with a membrane area of $2 \mathrm{~mm} \times 1 \mathrm{~mm}$. Polyimide was chosen as membrane material due to its low thermal conductivity. The chip included three resistors. One resistor was centered and used as heating element. It was surrounded by two temperature sensors placed up- and downstream of the heater. All elements were made of platinum due to a well defined TCR and resistance behavior.

The layout and fabrication process were already presented in a previous work [9].

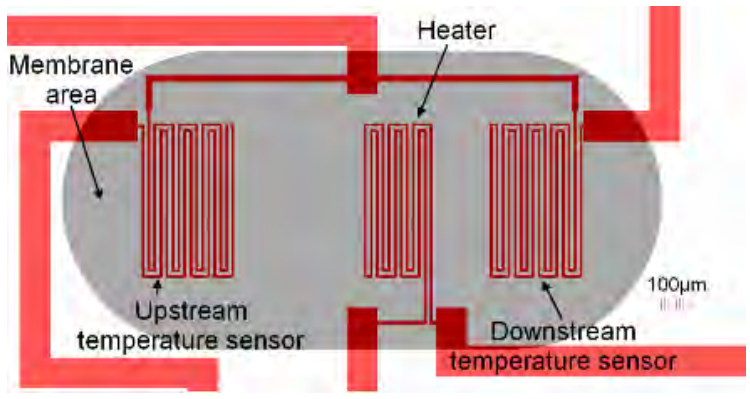

Fig. 1. Top view of sensor's membrane area. Upand downstream description marks the flow direction according to the heating element.

\section{Measurement Principle}

For flow measurements based on the hot wire principle the CCA or CTA are established procedures.
Both modes rely on the heater temperature, which decreases with an increasing flow speed. Heater temperature is either monitored through power or current consumption.

Constant amplitude of heat generation on the center resistor was achieved through a controller implemented in a LabVIEW program. The program enabled a default power with the simultaneous measurement of the heater temperature, which was changing due to a variation in flow and gas.

The electrical power of the heating element was set to $\mathrm{P}=5 \cdot(1+\sin (2 \cdot \pi \cdot f \cdot t))^{2}$, where $f$ is the oscillating frequency. It was adjusted to $f=1 \mathrm{~Hz}$.

The maximum temperature of the heating element is further investigated.

\section{Measurement Conditions}

The purpose of these experiments was to analyse the behavior of amplitude and phase shift under no flow condition and a volume flow between 10 to $200 \mathrm{sccm}$ (corresponding to a flow speed of $1.5 \mathrm{~m} / \mathrm{s}$ ). Several gases at different flow velocities were analyzed at room temperature.

The sensor was mounted in a gas chamber, where it was possible to change the gas concentration under no flow conditions. The gas concentration of an argon-helium mixture was changed stepwise from $100 \%$ argon to $100 \%$ helium.

To investigate the sensor's behavior under flow conditions, the sensor was built in a flow channel with a cross section of $2 \mathrm{~mm}^{2}$, which guaranteed a fully developed laminar flow profile (Reynolds number <270). The flow speed was set with a mass flow controller. Carbon dioxide, air and argon were used to evaluate the sensor performances.

\section{Results in no flow condition}

The maximum heater temperature was plotted versus the theoretical values of the gas' thermal diffusivity and gas' thermal conductivity. Figure 2 illustrates the heater temperature as a function of thermal diffusivity, Fig. 3 as a function of thermal conductivity. As it can be seen the heater temperature decreased significantly with an increase in thermal conductivity or diffusivity. The comparison between Fig. 2 and 3 shows that the temperature of the heater was strongly affected by the gas' thermal conductivity and not by its thermal diffusivity.

The increase of thermal conductivity by a factor 10 caused a heater temperature 
reduction of around $33 \%$. Curve fitting of the measured values was used to analyze the results. For fitting Table Curve [10] was utilized. The empirical correlation between thermal conductivity and maximum heater temperature can be given by

$$
\lambda=a+\frac{b}{T^{2}}
$$

where $\lambda$ is the gas thermal conductivity, $T$ the maximum heater temperature and $a, b$ curve fitting parameters. The coefficients $a$ and $b$ were calculated to $a=0.00428 \mathrm{~W} /(\mathrm{m} \cdot \mathrm{K})$ and $b=318.8(\mathrm{~W} \cdot \mathrm{K}) / \mathrm{m}$. The coefficient of determination $R^{2}$ was 0.998 . The fitted curve is indicated in Fig. 3.

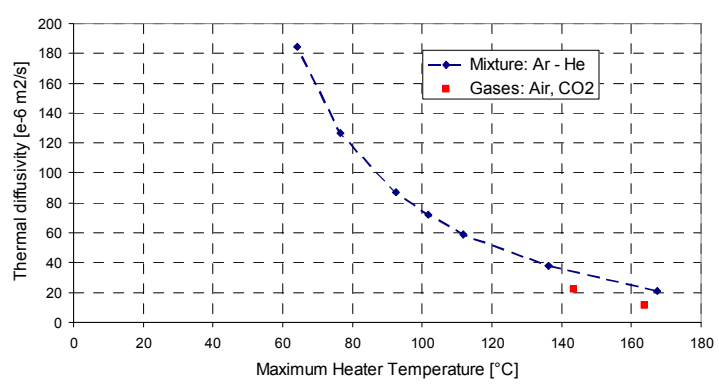

Fig. 2. Maximum value of heater temperature as function of thermal diffusivity in a non-flowing fluid Measurement results. Gas type was varied.

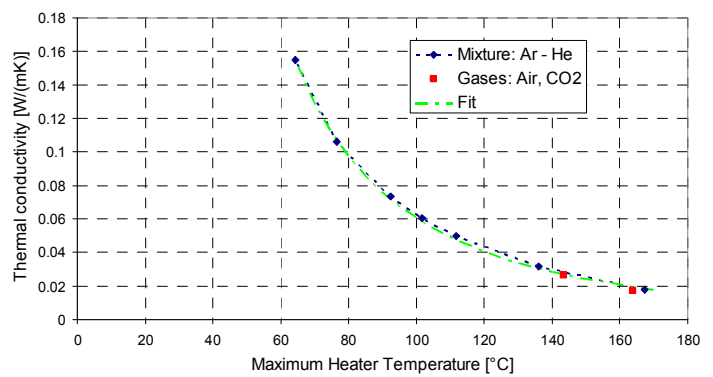

Fig. 3. Maximum value of heater temperature as function of thermal conductivity in a non-flowing fluid - Measurement results. Gas type was varied.

\section{Results in a flowing fluid}

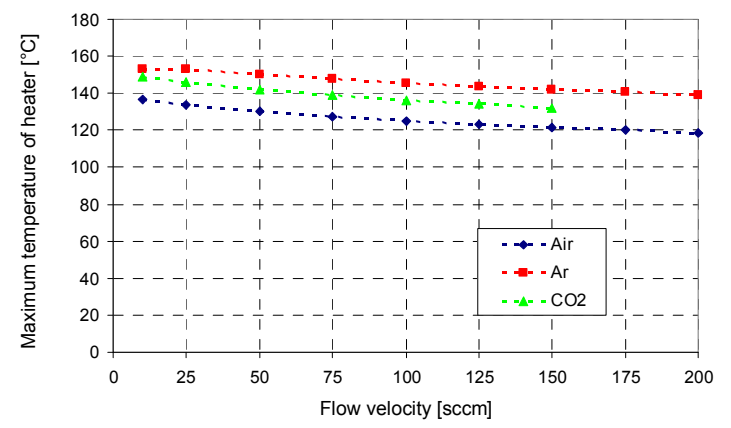

Fig. 4. Heater temperature as function of flow speed for the tested gases air, argon and carbon dioxide.
Figure 4 illustrates the decrease of the heater temperature with an increase in flow velocity, which was not linear. The figure implies that it can be clearly distinguished between the gases air and argon, but not between argon and carbon dioxide. The behavior of carbon dioxide and air was different although theses gases have only a difference of $5 \%$ in thermal conductivity (see Tab. 1). The detection of the heater temperature was sufficient to specify gas and flow range, if it was known that the gases which have to be analyzed show a significant difference in thermal conductivity.

\section{Simulation Model}

For varying the experimental results a 2D-Simulation model was implemented with COMSOL Multiphysics. The sensor geometry was drawn as described in the previous chapters. The platinum resistors were built with the highly conductive layer feature. The material constants for the used material platinum, polyimide and glass - were taken form the built-in materials library. The key physical parameters of the gases used in the simulations are summarized in Tab. 1. The reference temperature was set to $\mathrm{T}=25^{\circ} \mathrm{C}$. The excitation of the sensor was implemented according to measurement principle description using the time domain in COMSOL Multiphysics. The simulated gases covered the same range of thermal conductivity as the measurements. The Heat Transfer module was used for the simulations in order to obtain results in a non-flowing fluid.

\section{Simulation results}

The maximum heater temperature as function of the thermal conductivity for a non flowing fluid is given in Fig. 5. The results correlate to the experiments; where the lowest heater temperature corresponds to helium, the highest to argon. The simulation results were analyzed with equation (1). The fitting parameters $a$ and $b$ are obtained with table curve $(a=-$ $0.0051 \mathrm{~W} /(\mathrm{m} \cdot \mathrm{K}) ; \quad b=659.83(\mathrm{~W} \cdot \mathrm{K}) / \mathrm{m})$. The coefficient of determination $R^{2}$ is 0.999 .

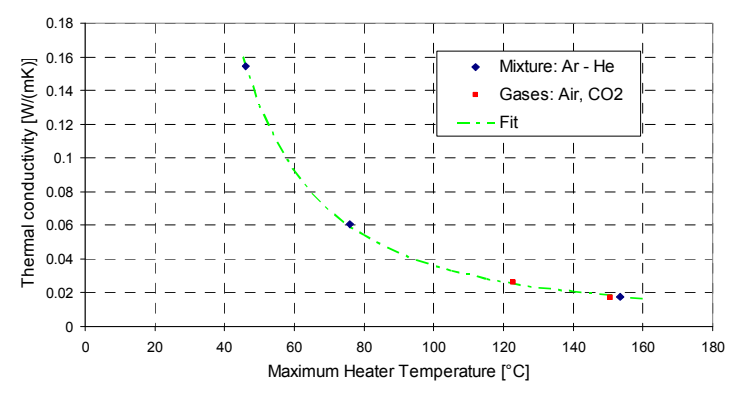

Fig. 5. Maximum value of heater temperature as function of thermal conductivity - Simulation results. 


\section{Discussion}

A comparison between simulations and experimental measurements show an accordance of $9 \%$ carbon dioxide and argon. Only an accordance of $18 \%$ is given for helium. These differences might be further reduced with an improvement of the simulation model. The optimization can be done with respect to buoyancy forces to cover the wide temperature range occurring of the high variation in thermal conductivity.

An estimation of the heat flux into the fluid can be obtained by the Prantl-, Grasshof- and Reynolds-number as explained in Ref. [11]. This estimation takes all thermal gas properties into account.

The presented measurements and simulations depicted that the dominant parameter were thermal conductivity and/or flow speed. Further experiments have to be carried out to find a more precise correlation. For instance a difference in the fluid density leads to a difference in thermal boundary layer thickness, which can be obtained by the Reynoldsnumber. This can be one evidence for the difference curve progression of carbon dioxide and argon in Fig. 4.

\section{Conclusions}

By means of measurements and simulations, it has been shown that it was possible to obtain simultaneously gas type and flow speed if only the temperature of the heater is evaluated. However, certain conditions had to be fulfilled or have to be given by the application. In this contribution, it has been shown that a high difference in thermal conductivity for the used gases has to be given. The difference of thermal conductivities was depending on the flow range which has to be covered. Furthermore, it was found out that thermal conductivity was the dominant parameter.

To achieve a more detailed resolution the upand downstream resistances have to be investigated as well; evaluating e.g. amplitude and phase shift. Some approaches are already given in Refs. [12, 13].

\section{References}

[1] Innovative Sensor Technology IST AG, Datasheet, Flow Sens FS5/FS5A, Thermal Mass Flow Sensor for all-purpose use in Gases, accessed January $13^{\text {th }}, 2013$, http://www.istag.com/eh/istag/resource.nsf/imgref/Download_DFFS5_E1.0.p df/\$FILE/DFFS5_E1.0.pdf

[2] F. Kohl, A. Jachimowicz, J. Steurer, R. Glatz, J. Kuttner, D. Biacovsky, F. Olcaytug, G. Urban, A micromachined flow sensor for liquid and gaseous fluids, Sensors and Actuators A:
Physical, Vol. 41, No. 1-3, 293-299 (1994); doi: 10.1016/0924-4247(94)80126-6

[3] M. Elwenspoek, Thermal flow micro sensors, Proc. Semiconductor Conference, 1999. CAS '99 Proc. 1999 International, Oct. 5-9, Sinaia, Romania, Vol. 422, 423-435 (1999); doi: 10.1109/SMICND.1999.810580

[4] A. Cubukcu, E. Zernickel, U. Buerklin, G. Urban, A 2D Thermal Flow Sensor with sub-mW power consumption, Sensors and Actuators A: Physical, Vol. 163, 449-456 (2010); doi:

10.1016/j.sna.2010.08.012

[5] NEL PPDS - Physical Properties of Fluids

[6] I. Simon, M. Arndt, Thermal and gas-sensing properties of a micromachined thermal conductivity sensor for the detection of hydrogen in automotive applications, Sensors and Actuators A: Physical, Vol. 97-98, 104-108 (2002); doi: 10.1016/S0924-4247(01)00825-1

[7] H. Ernst, A. Jachimowicz, G. Urban, Dynamic thermal sensor-principles in MEMS for fluid characterization, IEEE Sensors Journal, Vol. 1, No. 4, 361-367 (2001); doi: 10.1109/7361.983477

[8] A.S. Cubukcu, G. Urban, Simulation and fabrication of a 2D-flow sensor for simultaneous fluid characterization, Proc. of the Eurosensors XXIII Conference, Procedia Chemistry, Lausanne, Switzerland, Sept. 6-9, 887-890 (2009); 10.1109/SENSOR.2009.5285928

[9] C. Hepp, F. Krogmann, J. Polak, M. Lehmann, G. Urban, "AC Characterisation of Thermal Flow sensors with Fluid Characterisation Feature", Proc. of the $16^{\text {th }}$ Int. Conference on Solid-State Sensors, Actuators and Microsystems Transducers, Bejing, China, June 5-9, 2011, 1084-1087 (2011); doi:

10.1109/TRANSDUCERS.2011.5969180

[10] Table curve 2D - SYSTAT

[11] F. Incropera, D. DeWitt, T. Bergman, A. Lavine, "Fundamental of Heat and Mass Transfer", $6^{\text {th }}$ edition, John Wiley, 2007.

[12] C. Hepp, F. Krogmann, G. Urban, "Determination of thermal gas properties and flow speed using thermal flow sensors", Proc. of the $1^{\text {th }}$ Int. Conference Microfluidic Handling Systems, Enschede, The Netherlands, Oct. 10-12, 91-94(2012);

[13] D. Reyes, G. Urban, "Simulation of the oscillatory excitation of a thermal flow sensor", Proc. of the $1^{\text {th }}$ Int. Conference Microfluidic Handling Systems, Enschede, The Netherlands, Oct. 1012, 22-25 (2012); 